\title{
An Ontology-Based Well-Founded Proposal for Modeling Resources and Capabilities in ArchiMate
}

\author{
Carlos L. B. Azevedo ${ }^{1}$, Maria-Eugenia Iacob ${ }^{2}$, João Paulo A. Almeida ${ }^{1}$, \\ Marten van Sinderen ${ }^{3}$, Luís Ferreira Pires ${ }^{3}$ and Giancarlo Guizzardi ${ }^{1}$ \\ ${ }^{1}$ Ontology and Conceptual Modeling Research Group (NEMO), \\ Federal University of Espírito Santo (UFES), Vitória, ES, Brazil \\ ${ }^{2}$ Department of Industrial Engineering and Business Information Systems, \\ ${ }^{3}$ Information and Software Engineering Systems Group, Centre for Telematics and Information Technology, \\ University of Twente, Enschede, The Netherlands \\ clbazevedo@inf.ufes.br,m.e.iacob@utwente.nl,jpalmeida@ieee.org, \\ $\{$ m.j.vansinderen, 1.ferreirapires\}@utwente.nl, gguizzardi@acm.org
}

\begin{abstract}
The importance of capabilities and resources for portfolio management and business strategy has been recognized in the management literature and on a recent proposal to extend ArchiMate, which includes these concepts in order to improve ArchiMate's coverage of portfolio management. This paper presents an ontological analysis of the concepts introduced in that proposal, focusing in particular on the resource, capability and competence concepts. As a result, it proposes well-founded recommendations for improvements, enhancing its suitability and integration possibilities.
\end{abstract}

Keywords: Capability, Resource, Enterprise Architecture Modelling, Ontology-based Semantics, ArchiMate

\section{INTRODUCTION}

Constant shifts in markets, competition, technology and regulation drive organizations to promote changes, and to continuously adapt and improve their organizational structures and business processes. Failing to address these shifts affects organizational performance negatively [1]. A fundamental question in the field of strategic management is how organizations gain and sustain competitive advantages [2]. Research has shown that the average period during which organizations are able to sustain their competitive advantage has decreased over time [3]. This suggests that organizations have to build successive temporary advantages [4]. In order to be able to accomplish this, an organization needs to employ its resources and capabilities in the most effective and efficient manner. This observation is not only valid at the level of one organization, but also within business networks, in which organizations establish partnerships with the goal of pooling their capabilities and resources and improving their own inter-enterprise relationships. A major concern on strategic management focuses on improving the usage of organizational capabilities and resources, in order to improve performance, quality and to reduce costs [5] [6]. The suggestion that organizational resources and capabilities are key success factors for competitive advantage have been proposed already in the 90's [7] and is still a predominant topic [8].

In the work presented in [9], many organizations have been analyzed in order to answer the question "to what extent do access and changes to resource bases influence the development of dynamic capabilities in new firms?". The work provides statistical evidence for the relationship between organizational resources and the subsequent capabilities of the organization. Different resources lead to different capabilities, and the changes of resources over time have a great impact on organizations' capabilities [9].

Resources-centric theories regard an organization as a bundle of resources [10]. They suggest that the resources' properties (e.g., rare, valuable, non-substitutable or inimitable) confer organizations competitive advantage [11]. However, these works also observe that resources by themselves are not useful unless they are correctly employed [10]. Furthermore, the way they are used defines the outcome: [12] stated that "exactly the same resources when used for different purposes or in different ways and in combination with different types or amounts of other resources provide a different service or set of services".

Given the importance of capabilities and resources for the enterprise, an approach to integrate capabilities and resources in the discipline of Enterprise Architecture (EA) has been proposed in [13]. This approach entails the extension of the ArchiMate enterprise architecture modeling language [14] with Business Strategy and Valuation Concepts (BSVC). The BSVC includes the definition of the concepts of capability, resource, competence and risk. Its aim is to improve ArchiMate's portfolio management capabilities and to align it with the disciplines of business strategy and portfolio management. The various concepts are related to each other and to the ArchiMate core, and it is demonstrated how they could be used in enterprise modeling.

Similarly to other earlier ArchiMate extension proposals (e.g., the motivation extension [15]), the initial development of the BSVC has been conducted without a rigorous definition of the semantics of the proposed modeling elements. The absence of such definitions could lead to several modeling and communication problems. For example, when various modelers share a model, this could lead to the False Agreement Problem [16], in which each modeler would come to a different interpretation of the same model and would not be aware of the conflict. This would result in enterprise architecture models that cannot serve their purpose 
as tools for communication between stakeholders, and would decrease their value in the pursuit of informed decisionmaking.

In order to address this shortcoming, this paper discusses the semantics of the resource, capability and competence modeling elements, and proposes well-founded recommendations for improvements of the original proposal. We analyze and interpret the elements of the extension with respect to the Unified Foundational Ontology (UFO). The remainder of this paper is structured as follows. Section II describes the Business Strategy and Valuation Concepts extension briefly and introduces a running example. Section III introduces the ontological foundational concepts used in our analysis. Sections IV, V and VI provide interpretations for the concepts of capability, resource and competence, respectively. Each concept is interpreted according to the metamodel, the textual definitions, and the examples of usage provided proposed in [13], and in the related literature. Section VII summarizes our findings and postulates a number of improvement recommendations. Section VIII discuss related work. Finally, Section IX presents our conclusions and outlines future work.

\section{The Business StRategy AND VALUATION CONCEPTS EXTENSION}

The BSVC extension is based on the analysis and review of relevant business strategy and portfolio management literature [13]. For this reason, many concepts present in other approaches, such as [17] and [18] have been incorporated in the BSVC.

Table 1 shows the definitions and concrete syntax for the modeling elements introduced by the extension. Note that in this section we have preserved the definitions that were provided originally in [13]. These will be object of analysis in Sections IV, V and VI.

Table 1. Original definitions and syntax of BSVC constructs

\begin{tabular}{|l|l|c|}
\hline $\begin{array}{l}\text { Modeling } \\
\text { element }\end{array}$ & Definition & $\begin{array}{l}\text { Concrete } \\
\text { syntax }\end{array}$ \\
\hline Resource & $\begin{array}{l}\text { "an asset owned or controlled by } \\
\text { an individual or organization" }\end{array}$ & Resource \\
\hline Capability & $\begin{array}{l}\text { "the ability (of a static structure } \\
\text { element, e.g., actor, application } \\
\text { component, etc.) to employ } \\
\text { resources to achieve some goal" }\end{array}$ & Capability \\
\hline Competence & $\begin{array}{l}\text { "the definition of competence } \\
\text { [...] is almost identical with that } \\
\text { of personnel-based resources". }\end{array}$ & Resource \\
\hline
\end{tabular}

Figure 1 shows the metamodel fragment, as proposed in [13], for the integration of the BSVC with the ArchiMate core metamodel and its extensions [14].

The resource concept is prominently present in most valuation techniques, in business modeling approaches, and in constraint optimization models in which they are mathematically defined and constrained. This confirms the importance given to the concept in the management literature [13]. A resource represents an asset owned or controlled by an individual or organization. Resources are related to the

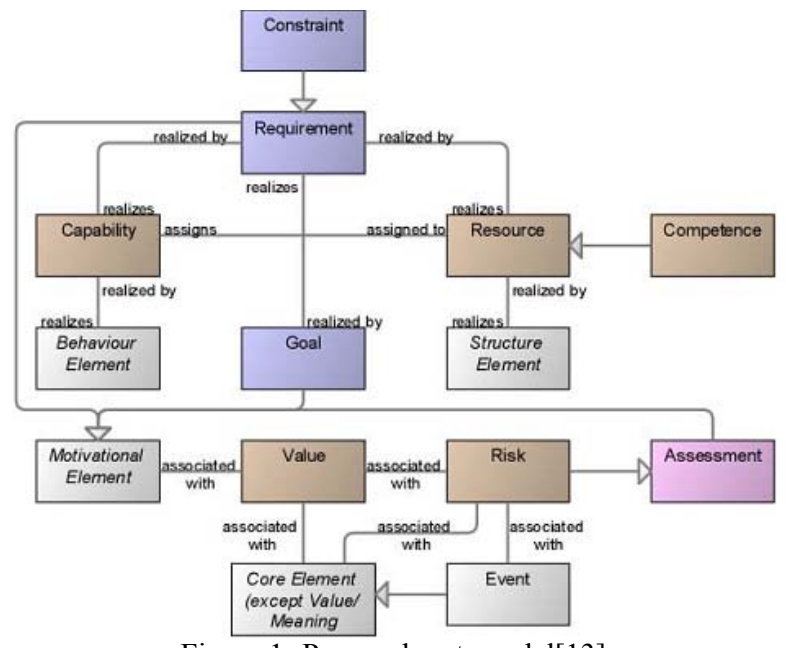

Figure 1- Proposal metamodel[13]

motivation extension, in particular to requirements and goals, through the realization relation. The argument for this relationship is the fact that goal achievement assumes availability and (constrained) consumption of certain resources. This view is based on the mathematical formulation of constrained optimization models, in which a goal function is minimized/maximized subject to a system of constraints (expressed as inequalities) imposed to the resources to be consumed for the achievement of the goal. Thus, a resource may realize a requirement, which in turn, may realize a goal. Furthermore, a resource is realized by structure elements, and is regarded in [13] as an abstraction of these elements.

Capability is defined as the ability (of a static structure element, e.g., actor, application component, etc.) to employ resources to achieve some goal. Similarly to resource, capability is regarded in [13] as an abstraction of some behavior. The assignment relationship between resource and capability expresses the ability to employ (i.e., configure, integrate, etc.) resources.

The competence concept is introduced in [13] as a specialization of resource based on definition of competence proposed by [19], which equates competence to personnelbased resources.

Figure 2 shows an ArchiMate model which we used as an example in this paper. The example focuses on the 'All Risks Insurance' product, and on the capabilities ('Claim Handling Capability', 'Insuring Capability', 'Selling Capability', etc.) associated with this product, The example also reviews the resources assigned to the capabilities ('Damage Assessment Resources', 'Money', 'Authorized Garage', etc.). As in any real business situation, in our example some restrictions may hold that constrain capabilities, and the usage of resources. We formulated them as requirements that must be satisfied: the Damage assessment team may not be larger than 20 full time employees, while the handling costs per claim should not be higher than $20 €$. The example shows that capabilities may be decomposed into other capabilities, and may be realized by some behavior elements (as in the case of 'Insuring Capability' and 'Claim Handling Capability'). Similarly, resources can be decomposed and are realized by structure elements. 


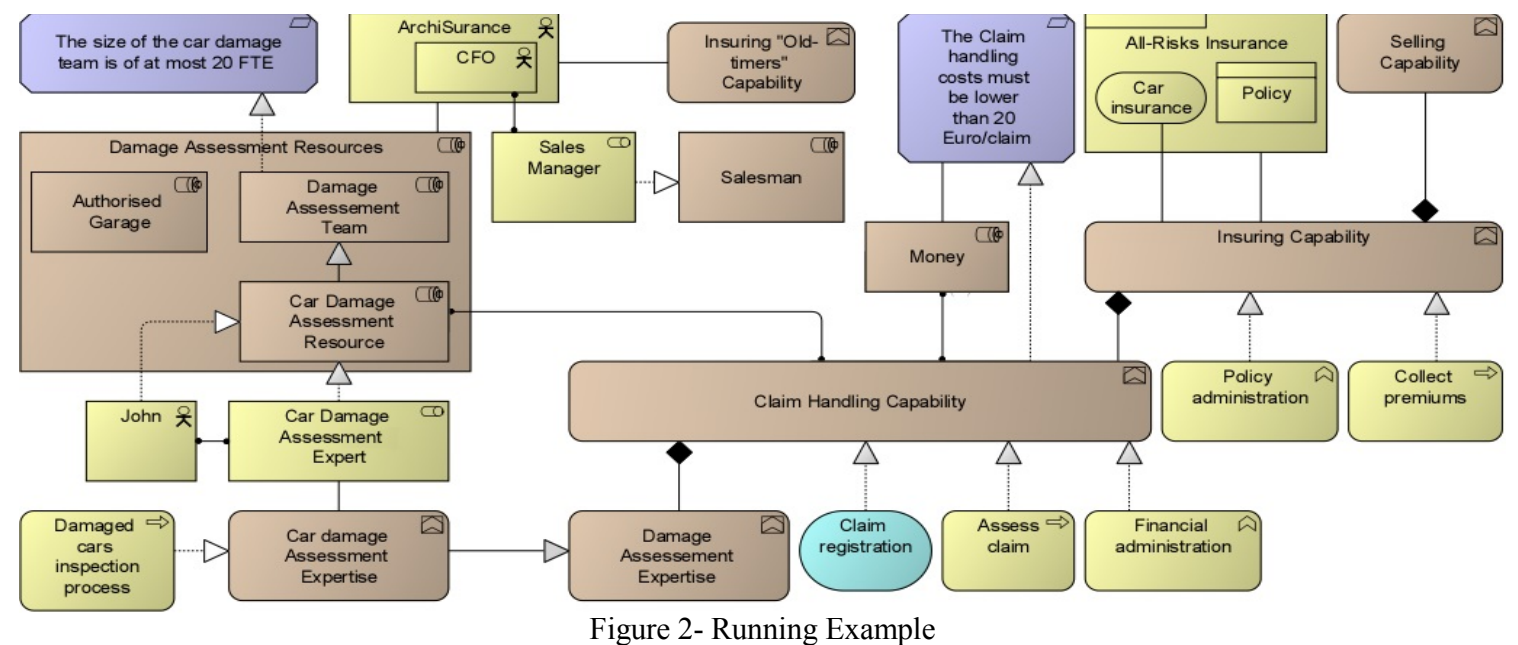

\section{ONTOLOGICAL FOUNDATIONS}

In our ontological analysis we make use of the Unified Foundational Ontology (UFO) as semantic foundation. The UFO foundational ontology has been previously used to analyze and interpret the semantics of the ArchiMate motivation concepts, having led to recommendations of that proposal that have been incorporated in the latest ArchiMate specification [15]. Further, UFO has been also employed in other semantic analyses, such as that of ARIS EPCs [20], i*/TROPOS [21], goals and business processes models [22] and role-related concepts in EA [23]. For a full discussion regarding this ontological foundation we refer to [24], and [25]. This section is based on the UFO description in [15].

A fundamental distinction in this ontology is between the categories of individuals and universals. Universals are predicative terms that can be applied to a multitude of individuals, capturing their general aspects. Individuals are entities that exist in reality possessing a unique identity and that can instantiate one or more universals.

Further, UFO makes a distinction between the concepts of endurants and events. Endurants are individuals that persist in time while keeping their identity, in the sense that if we say that in circumstance $c l$ an endurant $e$ has a property $p 1$ and in circumstance $c 2$ a property $p 2$ (possibly incompatible with $p 1$ ), e is the same endurant in each of these situations. Examples can include a particular person (say Peter) weighting $70 \mathrm{~kg}$ in one circumstance and $78 \mathrm{~kg}$ in a different circumstance, being the same individual (Peter) in all circumstances. Other examples include organizations (the University of Twente, the Federal University of Espírito Santo) and everyday objects (a ball, an apple, etc.). Events, in contrast, are individuals composed of temporal parts, they happen in time, in the sense that they extend in time and accumulate temporal parts. Examples include a particular execution of a business process, a meeting or a birthday party. Whenever an event occurs, it is not the case that all of its temporal parts necessarily occur. For instance, if we consider a business process "Buy a Product" at different time instants, at each time instant only some of its temporal parts are occurring.
A substantial is an endurant that does not depend existentially on any other individual, what is usually referred by the common sense term "object". In contrast with substantials, moments (also known as 'abstract particulars' and 'tropes' [26], [27]) are existentially dependent entities, i.e., for a moment $\mathrm{x}$ to exist, another individual must exist, named its bearer. Examples of moments include an apple's color, John and Mary's marriage, an electric charge on a conductor, etc. Moments include both qualities (e.g., color, weight, temperature) and dispositions (e.g., the fragility of a glass, the disposition of a magnet to attract metallic material). Dispositions are moments that are only manifested in particular situations, and that can also fail to be manifested. When manifested, they are manifested through the occurrence of events. Take for example the disposition of a magnet $m$ to attract metallic material. The object $m$ has this disposition even if it is never manifested, for example, because $m$ was never close to any magnetic material. Nonetheless, $m$ can certainly be said to possess that intrinsic property [27],[28].

Existential dependence can be used to differentiate intrin$\underline{\text { sic }}$ and relational moments. Intrinsic moments are dependent on a single individual, while relational moments (also called relators) depend on a plurality of individuals. Examples of the first include an apple's weight and color, while examples of the latter include John and Mary's marriage, John's enrollment at the University of Twente. A relator is the truthmaker of a material relation.

A universal is rigid if it necessarily applies to its instances, i.e., in every possible world (e.g., Apple, Person). A kind is the rigid substantial universal that supplies a principle of identity for substantial individuals that instantiate them. Every substantial individual must be an instance of exactly one kind. In contrast to rigid universals, a universal is antirigid if it does not apply necessarily to all its instances. Roles are anti-rigid and relationally-dependent universals (e.g., Student, Husband). This means that roles are played by a substantial whenever there is a relator connecting it to one or more other substantials or when it participates in an event playing what is called a processual role.

Whenever different kinds have similar properties they may be subsumed by substantial universals termed rigid mixins (e.g., Physical Object, Living Entity). Some mixins are 
anti-rigid and represent abstractions of common properties of roles. These are termed role mixins. An example of role mixin is 'customer', which can be played by 'persons' and 'organizations'.

An agent is a specialization of substantial individual, representing entities capable of bearing intentional moments. These include mental states, such as individual beliefs, desires and intentions. Intentionality should not be understood as the notion of "intending something", but as the capacity to refer to possible situations of reality [29]. Every intentional moment has an associated proposition which is called the propositional content of the moment. In general, the propositional content of an intentional moment can be satisfied (in the logical sense) by situations in reality. Every intentional moment has a type (belief, desire or intention). The propositional content of a belief is what an agent holds as true. Examples include one's belief that the Eiffel Tower is in Paris and that the Earth orbits around the Sun. A desire expresses the will of an agent towards a possible situation (e.g., a desire that Brazil wins the Next World Cup), while an intention expresses desired states of affairs for which the agent commits to pursuing (internal commitment) (e.g., John's intention of going to Paris to see the Eiffel Tower).

Actions are intentional events, i.e., events with the specific purpose of satisfying (the propositional content of) some intention of an agent. The propositional content of an intention is termed a goal. Only agents are said to perform actions [22], as opposed to non-agentive objects that participate (non-intentionally) in events.

Agents can be further specialized into physical agents (e.g., a person) and social agents (e.g., an organization). $\underline{\text { Social agents are further specialized into institutional agents }}$ and collective social agents. Institutional agents are composed of a number of other agents, exemplifying what is termed a functional complex. "The parts of a functional complex have in common that they all possess a functional link with the complex. In other words, they all contribute to the functionality (or the behavior) of the complex" [24]. In addition to institutional agents, UFO also acknowledges the existence of collective social agents, which are distinguished from institutional agents in that all its members play the same role in the collective.

Similarly to agents, non-agentive objects can be specialized into physical objects and social objects. A category of social objects of particular interest to us is that of normative description. Normative descriptions are social objects that create social entities recognized in that context. Examples of normative descriptions include a company's regulations and public laws. Examples of social entities that can be defined by normative descriptions include social roles (e.g., president, manager, sales representative), social role mixins (whose instances are played by entities of different kinds, e.g., customer, which can be played by persons and organizations), social agent universals (e.g., that of political party, education institution), social agents (e.g., the Brazilian Labour Party, the University of Twente), social object universals (e.g., currency) and other social objects (e.g., the US dollar) or even other normative descriptions (e.g., a piece of legislation). Normative descriptions are recognized by at least one social agent. Figure 3 shows a fragment of the specializations of individuals in UFO.

\section{Ontological Analysis of Resource}

In this section we discuss the ontological analysis and interpretation of the resource modeling element introduced in [13]. We discuss possible interpretations in terms of UFO and consider the consequences of the various interpretations to the usage of the language. The problems revealed here are labeled and are object of recommendations in Section VII.

The Oxford Dictionary defines resource as "a stock or supply of money, materials, staff, and other assets that can be drawn on by a person or organization in order to function effectively". In [13], a similar intuition is put forward when motivating the resource element in the BSVC extension: "the achievement of a goal assumes the availability and (constrained) consumption of certain resources". Further, resources are also characterized as "assets owned or controlled by an organization".

Since "assets" are (valuable) things, in a first examination, this characterization seems to suggest that resources represent specific individuals, such as business actors (e.g., in case of staff as resource) or business objects (agentive

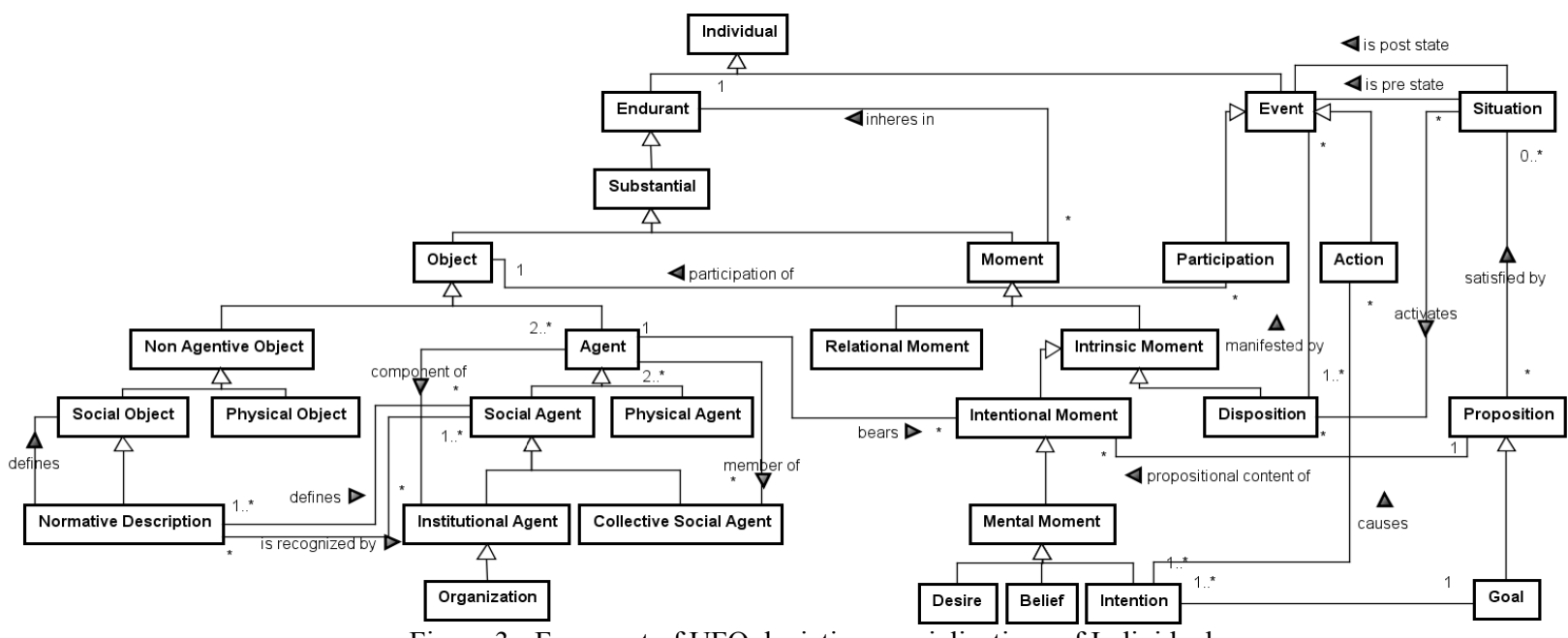

Figure 3 - Fragment of UFO depicting specializations of Individual 
objects (agents) or non-agentive objects). Nevertheless, this interpretation would show a clear case of construct redundancy [24], as the additional resource modeling element would serve no purpose, being supplanted by the previously existing structure elements of the language (such as business actor and business object). We must conclude this is not the intention of the designers of the extension, which indicate further that a resource is "an abstraction of structure elements" and include a "realizes" relation that may be used to connect structure elements to the resources they "realize". This suggests that it is not the specific structure element that is represented using a resource, but some more abstract notion, which reveals the dependence on a structure element with certain characteristics without specifying the particular element involved. In other words, we understand that the resource element defines some type of structure element (a universal), and that the structure element that realizes the resource instantiates this type. An example of this would be a model that includes a business actor 'John' that realizes a resource called 'Car Damage Assessment Resource'.

If we further consider that resources are used in the scope of the efforts to achieve goals, we can understand that a resource refers to the role an object (agentive or nonagentive) plays when employed in the scope of these efforts. In the example above, 'John' functions as a 'Car Damage Assessment Resource' in some context of the organization (for example that of the process of assessing damages).

Further, in order to play a particular role, an object may be required to instantiate some particular type (what is called an "allowed type" in [30].) For example, any "Car Damage Assessment Resource' may need to possess specific damage assessment skills, and thus instantiate some specific universal that is characterized by these skills.

Thus an intermediate conclusion is that resource represents an externally dependent universal (either a role or role mixin) that may be instantiated by objects of a particular allowed type. The fact that resource models both the role an object plays in a particular context of usage as well as its allowed type has some consequences to the terms used in the label of a resource. In some cases, such as 'Car Damage Assessment Resource', the context of usage is emphasized, focusing thus on the role or role mixin that is instantiated when the resource is used; in other cases, such as 'Money', the allowed type required for role playing is emphasized in the label.

An insight that comes out of this interpretation is that, as a role (mixin), a resource should have a context of usage, that in UFO is defined in the scope of a material relation (or in the scope of an event). This means some asset is a resource in a defined context, but not in others, e.g., the 'Car Damage Assessments Expert' is a resource in the 'Damaged cars inspection process' because of the 'Car Damage Assessment Expertise', but it should not be considered a resource for the 'Collect premiums' process. This is not directly expressible on the metamodel, because there is no notion of 'use' of resources. We label this as problem ' $\mathrm{R} 1$ '.

The resource concept is also defined as "an asset owned or controlled by an individual or organization". Being "owned or controlled" is understood as being available for the organization, e.g. by an employment contract between employers and employees, or by having the right/ownership over a certain object. For the cases in which the resource is an agentive element (agent) we understand the 'controlling' in the context of the social relator that bounds the particular individual or organization with the first, e.g., the employment contract. $A$ controls $B$ means that there is (possibly a set of) meta-commitment of $B$ towards $A$. In other words, $A$ has meta-claims over $B$ and, hence, the ability to delegate to $B$ and, consequently to increase its social ability [31]. For the interpretations in which the resource is a non-agentive object, we understand "the control or owning of the asset" as the ability to have that element participate in an event of interest, in which the organization $A$ has a certain right with respect to the object $O$ (for example a right to use, to consume, to destroy, to sell, etc). The current metamodel does not allow the modeler to identify who controls the resource (aside from the use of the very general and abstract associated with relation, which has no specific semantics). This could be an issue when there are multiple business actors (different organizations, business departments) that could control this resource. We call the lack of expressiveness of control relations ' $\mathrm{R} 2$ '.

Note further that the extension does not distinguish between resources potentially played by agents from those potentially played by non-agentive objects. We call this lack of expressiveness ' $\mathrm{R} 3$ '.

Other examples of usage indicate that not only business actors and objects may realize a resource, but also that business roles may be said to realize a resource. In our example the 'Car Damage Assessment Expert' business role realizes the 'Car Damage Assessment Resource'. In this case, we should understand that whichever object instantiates the role represented by the business role may also instantiate the role (mixin) represented by the resource. Intuitively in the example, not only 'John' but also any other damage assessment expert is a 'Car Damage Assessment Resource'. For these cases, the language does not settle whether one or more individuals instantiating the role (mixin) represented by the resource are required, used or controlled in the particular context. In other words, it is not possible to express whether all the instances of that type are required, used or controlled, if just one instance of that type is required, used or controlled or if an arbitrary set of instances of that type are required, used or controlled. We label this as problem 'R4'.

Further, the current ArchiMate language does not address the cases in which the resources are objects of interest or raw materials (e.g., 'Money' in the running example or gold, diamond, gas, etc), i.e., passive non-agentive elements. We believe the language designers have tried to cover this stretching the resource element, and using it directly to represent such objects. However, these would be resources that do not have any structure element to realize them, since there are no structure elements that can represent these types of objects in ArchiMate (the passive structure of ArchiMate focuses primarily on information objects). Also, in these cases, the resources are role (mixins) and the language will not be able to express if the same instance is to be considered a resource in various contexts (e.g. usage of the same 
amount of money in different contexts). Also, there is no possibility to express any property associated to the element itself (e.g., quantity of money, gold carats). We label this as problem 'R5'.

The resource concept is related to the motivation extension through the realization relation, in which a 'resource realizes a requirement'. According to [15], a requirement corresponds to a normative description, which states that if a system (in a broad sense) is to exist, then it must satisfy a particular proposition. In this case, we understand that proposition refers to the object (or objects) playing the resource role. Any instance instantiating the role (mixin) represented by the resource must satisfy the requirement's proposition. To put it simply, a requirement adds characteristics to a resource's allowed type.

The proposal also states that "the achievement of a goal assumes the availability and (constrained) consumption of certain resources". However, goals are not associated directly to resources and the proposal is silent on the issue of resource consumption. Resources and goals are only indirectly related through the 'goal is realized by requirement' and the 'requirement is realized by resource' relations. At this point, no interpretation can be given to the textual definition and further investigation would be required on the resources availability and consumption topic.

Now we focus on the common ArchiMate relationships that apply to the resource concept (specialization and aggregation). We interpret the specialization relation between resources as subsumption between the roles or (role mixins) represented by the resources. The aggregation relation between resources suggests some sort of whole-part relationship (aggregation in ArchiMate may be represented by containment, see in Figure 2 the relation between 'Damage Assessment Resources' as a whole and 'Authorised Garage', 'Damage Assessment Team' and 'Car Damage Assessment Resource'). Since resources may represent both agentive and non-agentive objects, it would be possible to combine these with aggregation. We interpret this as a very general sort of whole-part relation known as mereological sum. We must note the fact that there is no distinction between AND or OR resource aggregations in ArchiMate. Thus, when resources are aggregated, it is unclear whether all the aggregated resources are required/used, or whether one or any arbitrary number of them is required. We label this ' $R 6$ '.

\section{ONTOLOGICAL ANALYSIS OF CAPABILITY}

In this section we discuss the ontological analysis and interpretation of the capability modeling element introduced in [13]. Problems found by the analysis are labeled and are object of recommendations in Section VII.

The proposal defined capability as "the ability (of a static structure element, e.g., actor, application component, etc.) to employ resources to achieve some goal. [...] Also capability assumes the ability to employ (i.e., configure, integrate, etc.) resources". The definition also states that "capability (similarly to resource) can be seen as an abstraction of some behavior of the static structure element'.

We intuitively understand that a capability is attributed to some agent and gives that agent its power to bring about some behavior in order to achieve a desired outcome. From the excerpt "of a static structure element, e.g., actor, application component, etc.", the capability appears to belong to the specific individual that is to bring about the desired outcome. This would lead us to interpret that capabilities are dispositions in UFO. However, carefully examining the "abstraction of some behavior" fragment, and considering the same pattern that was employed by the language designers with respect to resources (as "abstractions of structural elements"), we understand that capabilities should be interpreted as types of dispositions (disposition universals in UFO).

Often a capability represents a general disposition type. For example, the 'car damage assessment expertise' capability is a general disposition type that is implicitly specialized into a more specific type (e.g. the capabilities to assess car damage produced by fire, to assess car damage caused by flood, to assess car damage after a crash).

This dispositional account is also applicable for cases in which the organization might hire a different company to perform processes realizing a capability and still state to have that capability (in this case because it has the disposition of delegating it [21], [31]). This is related to the idea of what an organization can "socially perform". If $A$ has a metacommitment from $B$ to execute $S$ then $A$ (socially) can do $S$. An object can have dispositions which arise from its parts (or from the network of its delegation relations [31]).

Preferably, the language should allow us to infer which individuals bear the dispositions that are related to that capability. However, the original metamodel does not include relations between the capabilities and the structure elements that are said to have the capabilities (such as a business actor or business role). As a consequence, the language does not allow one to identify the individuals or types of individuals which bear dispositions of the type presented by the capability (aside from using the generic and semantically-neutral association of ArchiMate). In other words, it is not possible to express in the language which structure element has a capability (including the capabilities an organization has), unless it is realized by some behavior element (we label this as 'problem C1'). For the case of resources, these are assigned to a capability (in the sense that they are used in order to leverage capabilities), but resources themselves do not have capabilities in the original extension. This is exemplified in Figure 2, in which the organization has not assigned the 'selling capability' to any resource. Since the language cannot express which are the capabilities beared by the 'Salesman' resource and the 'Sales Manager' structure element (business role), the organization is not able to know which resource or structure element has the required 'selling capability', to properly assign its performance.

The capability concept has three defined relationships according to the original metamodel. We now focus on "capability realizes requirement". Again, according to [15], a requirement corresponds to a normative description, which states that if a system (in a broad sense) is to exist, then it must satisfy a particular proposition. In this case, we understand that proposition refers to the dispositions that instantiate 
the disposition universal represented by the capability. The dispositions must be in accordance to the requirement, in order to satisfy its proposition.

We now focus on the relationships 'capability realized by behavior element' and 'resource assigned to capability'. We understand that the first needs to be considered also with the participation of the resource (via the 'resource assigned to capability' relationship). We understand that this pattern of relations can have two different interpretations (we label this as 'problem $\mathrm{C} 2$ '). A first one is that the resource object has a disposition that instantiates the disposition universal represented by the capability, and that the participation of the resource manifesting its disposition is required in order to perform the behavior element (an event universal), e.g., the capability 'Car damage Assessment Expertise' on Figure 2 is to be manifested in the 'Damaged cars inspection process' business process in order for the organization to perform that process. The second possible interpretation is that the capability is acquired (by the resource) with the performance of the behavior element [28], i.e., the resource acquires a capability after the process is performed e.g. The 'Car damage Assessment Expertise' capability is acquired with the occurrence of the 'Damaged cars inspection process' process. In UFO, this can be interpreted as that, $s$ is a situation in which the object has the disposition $d$ and $e$ is an event representing the behavior element, $e$ is a pre-state of $s$. If no resource is represented, the object that is acquiring or manifesting the capability is unknown (we label this as 'problem C3'). In this case, one can argue that it is an organization's capability, but it is not possible to clearly define it without relating it to the object and the specification does not define this case. Even when related to the resource concept, since the resource that is acquiring the disposition represents a universal, a type element, the actual object that is acquiring the disposition is undetermined. The language is not expressive enough to state if one individual, all the individuals that instantiates the universal or an arbitrary combination of individuals instantiating the universal are acquiring the disposition. In both in interpretations it is not possible to know in advance if it is one individual, all the individuals that instantiates that universal or an arbitrary combination of them that are related to the disposition. In the first interpretation, is not possible to know how many objects are to manifest their dispositions in the event represented by the behavior element. In the later, it is not possible to know which object is to acquire the disposition. We label this as 'problem C4'.

Now we focus on the common ArchiMate relationships, that apply to the capability concept. The specialization relation between capabilities should mean that a disposition universal (type) subsumes other disposition universal and the aggregation relation between capabilities is interpreted as (complex) dispositions, that are dispositions based on other dispositions [28]. We must note the fact that there is no distinction between AND or OR capability aggregations in ArchiMate. The language lacks expressiveness to state optional capabilities. The language also lacks expressiveness to state if all the capabilities associated to a behavior element are acquired or manifested, if just one of them is acquired or manifested or if an arbitrary number of them are acquired or manifested. We label this as 'problem C5'.

\section{ONTOLOGICAL ANALYSIS OF COMPETENCE}

In this section we discuss the ontological analysis and interpretation of the competence modeling element introduced in [13]. Problems found by the analysis are labeled and are object of recommendations in Section VII.

The competence concept was introduced in [13] as a "specialization of resource (intangible or personnel-based)". The proposal states that "a core competence is a particular strength of an organization. Core competences are the collective learning in organizations, and involve how to coordinate diverse production skills and integrate multiple streams of technologies. Examples of core competences include technical/subject matter know-how, a reliable process and/or close relationships with customers and suppliers". This was "based on the fact that the definition of competence [...] is almost identical with that of personnelbased resources". Personnel-based resources have been exemplified as "technical know-how, other knowledge assets including organizational culture, employee training, [...]".

Based on the facts above, we understand that a competence is something that an element, when provoked, is able to do, or to perform. It addresses the element's capacity of performing an activity. At a first examination, this characterization seems to suggest that competence is to be applied to specific individuals, and that it would be the disposition that inheres in the individual. However, the original metamodel shows that competence is a resource, that is a universal. Based on the resource interpretation, as a universal, we conclude that the competence also represents a universal, which would be a disposition type, whose instances inhere in the objects that play the role represented by a resource.

Since a competence is a specialization of resource, it inherits the resource's relations. We now focus on the 'competence realizes requirement'. According to requirement interpretation [15], we interpret this relation as: the disposition of the object satisfies the requirement's proposition. We understand that the competence is of the same disposition type as of the capability that is manifested (or acquired depending on the given interpretation) with the performance of the behavior element (event). The interpretation varies according to the interpretation given to the capability relationship (we label this as 'problem C2.1' since it is a consequence of C2). This can be interpreted as that: (i) by being able of executing a certain behavior element (an event universal), the resource object has a disposition $d$ (of the competence defined type) that instantiates the type represented by the capability or; (ii) that $s$ is a situation in which the object has the disposition $d$ and $e$ is an event representing the behavior element, $e$ is a pre-state of $s$, in which the $d$ is an instance of the competence. The relation 'competence is realized by structure element' relates the dispositions to the elements that bears them. This is interpreted in UFO as that the object that represents the structure element bears a disposition of that disposition type. This is desirable, however, since it is not enforced by the language, 
we label the lack of the knowledge on which object bears the disposition as 'problem $\mathrm{C} 1.1$ ' since it is associated to $\mathrm{C} 1$.

The interpretation of the competence concept points to the same ontological construct as the interpretation of the capability concept (we label this as 'problem C6'). The competence concept appears to have been introduced to fill the gap in the proposal that it is unknown which capabilities a resource (or structure element) has. This shows a case of construct redundancy in the language. According to [32], "construct redundancy occurs when more than one grammatical construct can be used to represent the same ontological construct". Our analysis confirms and explicates the informal suspicions raised in the original proposal text when it states that "depending on the (interpretation of the) definition of competence, one may argue that, for example it is more natural to introduce competence in the metamodel as a specialization of a capability" [13]. The original proposal also stated that "the semantic distance between competence, on one hand, and either resource or capability, on the other hand, is too small".

\section{ONTOLOGY-BASED RECOMMENDATIONS}

In this section we propose improvements to the language according to the problems found during the ontological analysis.

\section{A. Resource concept}

Problem R1 - Since they represent roles (or role mixins) that objects may play, a resource should have a context. An element is a resource in a defined context, but it is not a resource in all situations. This is not enforced in the language, because there is no notion of 'use' of resources.

The relation between resources and capabilities should be enforced, to have the meaning of contextualizing the usage of the resource (e.g., 'Money' is allocated to 'Claim handling capability'). This solution should be in accordance with the solution to Problems C2 and C2.1.

Problem R2 - The current metamodel does not allow the modeler to identify who controls the resource. Representing who controls the resource would allow for a finer grained modeling of resource ownership, e.g., in the case when multiple organizations or business departments control different resources.

Problem R3 - The language does not distinguish resources that are realized by agents from those realized by non-agentive objects. This is a lack of expressiveness that could be addressed by specializing resource.

Problem R4 - For the cases in which a resource is realized by a business role (a universal), the language does not settle whether one or more individuals instantiating the role (mixin) represented by the resource are required in the context of usage. The addition of cardinality constraints in the language can solve this problem. In fact, the lack of such constraints has being experienced by industry and some ArchiMate tools already implement a replication attribute for similar purposes.

Problem R5 - The ArchiMate language does not address the cases in which resources are passive non-agentive elements (objects of interest or raw material, e.g., 'Money', gold, diamond, gas, etc.) These resources do not have any structure element to realize them, since there are no structure elements' concepts that can represent these types of objects in ArchiMate. This implies that it is not possible to express if the same passive non-agentive object is to be considered a resource in various contexts. Also, there is no possibility to express any property associated to the object itself.

The ArchiMate language should add constructs to define passive non-agentive objects of interest, such as those mentioned above. This would expand the reach of the language's passive structure beyond information objects.

Problem R6 - There is no distinction between AND or OR resource aggregations in ArchiMate. The language lacks the expressivity to state optional resources. When the resource modeling element represents a pool of elements, the language does not define if all the resources on the pool are to be used, just one of them or any arbitrary number of them.

The ArchiMate language should (i) add distinctions between AND and OR aggregations; or (ii) add cardinality contraints in ArchiMate, in order to solve this problem.

These recommendations, if implemented, would improve the language and allow the specification of number of resources required to perform an activity, the specification of which resources are to perform behavior elements based on their intrinsic capabilities, the ability to model objects of interest and raw material as resources (passive non-agentive objects), to define which resources are required in a pool of resources and to distinguish between mandatory and optional resources.

\section{B. Capability and Competence concepts}

Problem C1 and C1.1 - The metamodel does not allow relations between the capabilities and the structure elements or the resources [individual or instances of the role (mixin)] that have those capabilities. Even for resources, the resources are assigned to manifest a capability, but resources have no capabilities. Consequently, it is not possible to express in the language which structure element bears dispositions of the type represented by a capability nor the capabilities the organization has, unless it is being realized by some behavior element.

A relationship should be added to the metamodel to express the capabilities of a structure element. The organization would then be able to select appropriately the structure element to be a resource in a behavior element based on its capabilities. The organization would also be able to know all the capabilities it can perform.

Problems C2 and C2.1 - The pattern of relationships on 'capability realized by behavior element' and 'resource assigned to capability' relations have two different ontological interpretations. As a consequence, the 'competence assigned to capability' also has two different interpretations.

The proposal should clearly state the desired interpretation. If both interpretations are desirable, there should be two different relations to state them.

Problem C3 - If no resource is represented, the object manifesting or acquiring the capability is unknown at the 'capability realized by behavior element' and 'resource assigned to capability' relationships pattern. 
The language should explicitly define the element manifesting or acquiring the capability. A solution would be to enforce a relation between the capability and the resource manifesting or acquiring it. It would also be possible to adopt a default referring to the organization being modeled, when no explicit relation is used.

Problem C4 - The language is not expressive to state the number/amount of resources that are related to the capabilities. For example, it is not possible to know how many instances of resources are to manifest their capabilities to perform a behavior element.

Similar to the solution to R4, a cardinality constraint addition in the language can solve this problem.

Problem C5 - There is no distinction between AND or OR capability aggregations in ArchiMate. The language lacks expressiveness to state optional capabilities. The language also lacks expressiveness to state if all the capabilities associated to a behavior element are manifested (or acquired), if just one of them is manifested (or acquired) it or if an arbitrary number of them are manifested (or acquired).

Similar to the solution to R6, the ArchiMate language should (i) add distinctions between AND and OR aggregations; or (ii) add cardinality constraints in ArchiMate.

Problem C6 - The interpretation of the competence concept points to the same ontological concept as the capability concept, which would be interpreted as construct redundancy. So, at first examination, the competence construct should be eliminated, and in any case, competence should no longer specialize resource. We nevertheless envision the possibility to specialize capabilities into those that can be related to agents (which could be competences) and those that can be related to non-agentive objects.

These recommendations, if implemented, would improve the language and make it able to express the capabilities a structure element has, allow the organization to assign resources to behavior elements when they have the required capabilities, express optional capabilities, eliminate the construct redundancy from the language leading to a more parsimonious language and clarifying the context of usage of resources, both for manifestation or acquisition of capabilities as to assignment of resources to behavior elements.

\section{RELATED WORK}

Some recent approaches have addressed the use of capabilities and resources on business strategy in EA. The DoDAF framework [33] has a viewpoint for capabilities. It models capabilities in order to catalogue and combine them to support business activities and services. In [34], TOGAF has been extended to support the modeling of the capabilities a Business Component (BC) can perform. A BC is a business unit that encompasses a set of activities, supported by assets including people, processes and technology. The approach uses capabilities as "an idealized conceptual structure that describes what a BC can do to create value for customers".

In recent years, a number of enterprise modeling approaches have been subject to ontology-based analysis. In [35], the authors performed an ontological analysis to the Business Process Modeling Notation (BPMN). Nine ontological deficiencies related to modeling when using the BPMN were found. In [20], the authors have defined the semantics of the ARIS framework concepts and relationships in terms of UFO. Problems regarding the ARIS Method were exposed and possible solutions to these problems were proposed. [15] performed an ontological analysis of the ArchiMate motivation extension proposal, unveiling problems and proposing improvement recommendations. To the best of our knowledge there are no comparable analyses addressing the modeling of capabilities and resources.

\section{CONCLUSIONS AND FUTURE WORK}

In this paper, we have discussed an ontological analysis of the BSVC ArchiMate extension and the associated notions of capability, resources and competences. We have employed a comprehensive foundational ontology which incorporates concepts to deal with objects, relations, roles, events, dispositions, as well as social and intentional concepts. Our main aim has been to clarify the semantics of the proposed modeling constructs, which should contribute to the application of the language in practice as a communication tool for stakeholders involved in decision making.

We have been able to clarify that the resource element represents a type-level entity, capturing the role of a (agentive or non-agentive) object in a particular context of usage. Our well-founded recommendations should lead to a language that would allow improved resource planning (as it would allow the specification of the context of usage of resources, the specification of the number/amount of resources required to perform an activity, the distinction between mandatory and optional resources) and would cover a broader spectrum of resources (with the ability to model non-agentive objects of interest and raw material as resources).

By considering capabilities as a type of dispositions, we have been able to account for what it means for a behavior element to realize a capability. Our recommendations should lead to a language that would allow improved resource and capability oversight (as it would allow one to relate capabilities and structure elements that possess capabilities, as well as relate resources with capabilities).

By considering that competences also should be interpreted as representing types of capabilities, we have been able to diagnose a case of construct redundancy. We have traced the root of the problem to a lack of relations to express the capabilities of resources. Our recommendations seem to lead to a more regular and parsimonious solution for the expression of human resources and their capabilities, which was not fully addressed with the introduction of the competence construct.

Similarly to what was discussed in [15] concerning the analysis of the motivation extension to ArchiMate, we do not intend to suggest that the terminology used in this paper should replace the terminology currently used in ArchiMate, and we do not intend to imply that the UFO conceptualization should be exposed directly to users of the standard. The main role of the ontological analysis has been to provide us with a rigorous framework to analyze the BSVC proposal. In this sense, the ontological analysis can be seen as a tool for hypothesis formulation, and the recommendations that we 
have identified here using ontological analysis should be considered as subject to further examination; for example, considering the pragmatic impact of amendments on the set of standards and its users. We have outlined the recommendations raised by the ontological analysis performed here and we believe that they can have direct application in the revision of the proposed metamodel before it reaches the standardization effort.

In our future efforts, we will investigate the relation between the resource element and the value element. The latter deserves careful attention from the perspective of semantic definition and ontological analysis, given its subjective nature and the flexibility in usage.

\section{ACKNOWLEDGMENTS}

This research is funded by CAPES, FAPES (PRONEX Grant $52272362 / 11,59971509 / 12$ and 601641131/12) and $\mathrm{CNPq}$ (Grants 310634/2011-3 and 311578/2011-0).

\section{REFERENCES}

[1] P. G. Audia, E. A. Locke, and K. G. Smith, "The paradox of success: An archival and a laboratory study of strategic persistence following radical environmental change.," Academy of Management Journal, vol. 43, no. 5, pp. 837-853, 2000.

[2] I. Barreto, "Dynamic Capabilities: A Review of Past Research and an Agenda for the Future," Journal of Management, vol. 36, no. 1, pp. 256-280, Dec. 2009.

[3] R. R. Wiggins and T. W. Ruefli, "Schumpeter's ghost: Is hypercompetition making the best of times shorter?," Strategic Management Journal, vol. 26, no. 10, pp. 887-911, Oct. 2005.

[4] K. M. Eisenhardt and J. A. Martin, "Dynamic capabilities: what are they?," Strategic management journal, vol. 21, no. 10-11, pp. 11051121 , Oct. 2000

[5] G. Ray, J. B. Barney, and W. A. Muhanna, "Capabilities, business processes, and competitive advantage: choosing the dependent variable in empirical tests of the resource-based view," Strategic Management Journal, vol. 25, no. 1, pp. 23-37, Jan. 2004.

[6] T. S. Baines, H. W. Lightfoot, O. Benedettini, and J. M. Kay, "The servitization of manufacturing: a review of literature and reflection on future challenges," Journal of Manufacturing Technology Management, vol. 20, no. 5, pp. 547-567, 2009.

[7] J. Barney, "Firm resources and sustained competitive advantage," Journal of management, vol. 17, no. 1, pp. 99-120, 1991.

[8] C. C. E. Helfat and S. G. S. Winter, "Untangling Dynamic and Operational Capabilities: Strategy for the (N) ever-Changing World," Strategic Management Journal, vol. 32, no. 11, pp. 1243-1250, 2011.

[9] A. McKelvie and P. Davidsson, "From Resource Base to Dynamic Capabilities: an Investigation of New Firms," British Journal of Management, vol. 20, pp. S63-S80, Mar. 2009.

[10] R. M. Grant, "Toward a knowledge-based theory of the firm," Strategic management journal, vol. 17, pp. 109-122, 1996.

[11] M. A. Peteraf and J. B. Barney, "Unraveling the resource-based tangle," Managerial and decision economics, vol. 24, no. 4, pp. 309323, 2003.

[12] T. Penrose, The theory of the growth of the firm. Oxford University Press, 1959.

[13] M.-E. Iacob, D. A. C. Quartel, and H. Jonkers, "Capturing Business Strategy and Value in Enterprise Architecture to Support Portfolio Valuation," 2012 IEEE 16th International Enterprise Distributed Object Computing Conference, pp. 11-20, Sep. 2012.

[14] M.-E. Iacob, H. Jonkers, M. M. Lankhorst, E. Proper, and D. A. C. Quartel, “ArchiMate 2.0 Specification: The Open Group," 2012.
[15] C. L. B. Azevedo, J. P. A. Almeida, M. van Sinderen, D. A. C. Quartel, and G. Guizzardi, "An Ontology-Based Semantics for the Motivation Extension to ArchiMate," 2011 IEEE 15th International Enterprise Distributed Object Computing Conference, pp. 25-34, Aug. 2011.

[16] N. Guarino, "Formal Ontology and Information Systems," no. June, pp. 3-15, 1998.

[17] J. Gordijn and H. Akkermans, "Designing and Evaluating E-Business Models," IEEE Intelligent Systems, vol. 16, no. 4, pp. 11-17, 2001.

[18] F. Zandi and M. Tavana, "A multi-attribute group decision support system for information technology project selection," International Journal of Business Information Systems, vol. 6, no. 2, pp. 179-199, 2010

[19] C. K. Prahalad and G. Hamel, "The core competence of the corporation," Resources, firms, and strategies: A reader in the resource-based perspective, pp. 235-256, 1990.

[20] P. S. Santos Jr, J. P. A. Almeida, and G. Guizzardi, "An ontologybased semantic foundation for ARIS EPCs," in Proceedings of the 2010 ACM Symposium on Applied Computing, pp. 124-130, 2010.

[21] R. Guizzardi and G. Guizzardi, "Ontology-based transformation framework from TROPOS to AORML," Social Modeling for Requirements Engineering, Cooperative Information Systems Series, pp. 547-570, 2011.

[22] E. C. S. Cardoso, P. S. Santos Jr, J. P. A. Almeida, R. S. S. Guizzardi, and G. Guizzardi, "Semantic Integration of Goal and Business Process Modeling," in International Conference on Research and Practical Issues of Enterprise Information Systems (CONFENIS 2010), 2010.

[23] J. P. A. Almeida, G. Guizzardi, and P. S. Santos Jr, "Applying and extending a semantic foundation for role-related concepts in enterprise modelling," Enterprise Information Systems, vol. 3, pp. 253-277, 2009.

[24] G. Guizzardi, "Ontological foundations for structural conceptual models," University of Twente, 2005.

[25] G. Guizzardi, R. Falbo, and R. S. S. Guizzardi, "Grounding software domain ontologies in the unified foundational ontology (ufo): The case of the ode software process ontology," in Proceedings of the XI Iberoamerican Workshop on Requirements Engineering and Software Environments, pp. 244-251, 2008

[26] E. J. Lowe, The four-category ontology: a metaphysical foundation for natural science. Clarendon Press, 2006.

[27] J. Heil, From an ontological point of view. Clarendon Press Oxford, 2003.

[28] G. Molnar, Powers: A study in metaphysics. Oxford University Press, 2006.

[29] J. R. Searle, Intentionality: An essay in the philosophy of mind. Cambridge University Press, 1983.

[30] C. Bock and J. Odell, "A more complete model of relations and their implementation: Roles," Journal of Object Oriented Programming, vol. 11, pp. 51-54, 1998.

[31] A. C. O. Bringuente, R. A. Falbo, and G. Guizzardi, "Using a Foundational Ontology for Reengineering a Software Process Ontology,” 2010.

[32] R. Weber, Ontological foundations of information systems. Coopers \& Lybrand and the Accounting Association of Australia and New Zealand Melbourne, 1997.

[33] D. A. Dryer, T. Bock, M. Broschi, and T. D. Beach, "DoDAF limitations and enhancements for the Capability Test Methodology," in Proceedings of the 2007 spring simulation multiconference Volume 3, pp. 170-176, 2007.

[34] T. Barroero, G. Motta, and G. Pignatelli, "Business capabilities centric enterprise architecture," in Enterprise architecture, integration and interoperability, pp. 32-43, Springer, 2010.

[35] J. Recker, M. Indulska, M. Rosemann, and P. Green, "The ontological deficiencies of process modeling in practice," European Journal of Information Systems, vol. 19, no. 5, pp. 501-525, Jun. 2010. 